\title{
Pediatric B-lymphoblastic leukemia with RUNX1 amplification: clinicopathologic study of eight cases
}

\author{
Kaaren K Reichard ${ }^{1}$, Huining Kang ${ }^{2}$ and Sheldon Robinett ${ }^{3}$ \\ ${ }^{1}$ Department of Pathology, University of New Mexico, Health Sciences Center, Albuquerque, NM, USA; \\ ${ }^{2}$ Department of Internal Medicine, University of New Mexico, Health Sciences Center, Albuquerque, NM, \\ USA and ${ }^{3}$ TriCore Reference Laboratories, Albuquerque, NM, USA
}

\begin{abstract}
B-lymphoblastic leukemia (a.k.a. precursor B-cell acute lymphoblastic leukemia) is a heterogeneous disease at the clinical, morphologic, immunophenotypic and genetic levels. Recurrent genetic abnormalities in B-lymphoblastic leukemia with prognostic significance are well known and specifically delineated in the WHO 2008 classification (eg hyperdiploidy, $\mathrm{t}(9 ; 22)(\mathrm{q} 34 ; \mathrm{q} 11.2) ; B C R-A B L 1, \mathrm{t}(12 ; 21)(\mathrm{p} 13 ; q 22) ; E T V 6-R U N X 1)$. In recent years, a subgroup of B-lymphoblastic leukemia with the recurring genetic alteration of RUNX1 amplification has emerged. This subgroup has a low incidence $(2 \%)$ and an increased risk of relapse and overall worse outcome. Given these apparently distinctive clinicopathologic features, we evaluated eight cases of pediatric B-lymphoblastic leukemia with RUNX1 amplification treated on Children's Oncology Group protocols from 2000-2009. Compared with 25 consecutive B-lymphoblastic leukemia cases without RUNX1 amplification, we identified a trend toward male predominance $(P$-value $=0.082)$ and low white blood cell count at presentation $(P$-value $=0.081)$ in B-lymphoblastic leukemia with $R U N X 1$ amplification. Older age at presentation was significant (median age 9.5 years, $P$-value $=0.006$ ). There was no significant difference in the presence of central nervous system disease, CD20 or myeloid antigen positivity on the blasts or percent circulating blasts in B-lymphoblastic leukemia with RUNX1 amplification versus other B-lymphoblastic leukemia types. Seven of eight patients (88\%) are alive and free of disease at the time of last checkup (mean 50 months, range 14-116 months). Although we see a relatively good outcome in our small cohort of patients, recent findings from the Children's Oncology Group on a large set of patients suggests otherwise that these patients may have an inferior outcome compared with patients with B-lymphoblastic leukemia without RUNX1 amplification. Longterm follow-up in larger cohorts including minimal residual disease correlation is required.

Modern Pathology (2011) 24, 1606-1611; doi:10.1038/modpathol.2011.118; published online 5 August 2011
\end{abstract}

Keywords: acute leukemia; $A M L 1$; amplification; B-lymphoblastic leukemia; precursor B-cell; RUNX1

B-lymphoblastic leukemia (a.k.a. precursor B-cell acute lymphoblastic leukemia) is a heterogeneous disease at the clinical, morphologic, immunophenotypic and genetic levels. Despite modern therapies targeted against a combined risk assessment of age, white blood cell count, central nervous system involvement, early/slow treatment response, genetics and minimal residual disease, $\sim 20 \%$ of

chi-

Correspondence: Dr KK Reichard, MD, Department of Pathology, University of New Mexico, 1001 Woodward Place NE, c/o TriCore Reference Laboratories, Albuquerque, NM 87102, USA.

E-mail: kreichard@salud.unm.edu

Received 1 April 2011; revised 20 May 2011; accepted 31 May 2011; published online 5 August 2011 ldren experience disease recurrence. Continued refinement of therapeutic protocols and risk-stratification categories are necessary for further improving B-lymphoblastic leukemia response rates.

Recurrent genetic abnormalities in B-lymphoblastic leukemia with prognostic significance are well known and are specifically categorized in the WHO 2008 classification. ${ }^{1}$ These distinct genetic subgroups of B-lymphoblastic leukemia include hyperdiploidy, hypodiploidy, t(9;22)(q34;q11.2); BCR-ABL1, $\mathrm{t}(\mathrm{v} ; 11 \mathrm{q} 23) ; M L L$ rearranged, $\mathrm{t}(12 ; 21)(\mathrm{p} 13 ; \mathrm{q} 22) ; E T V 6-$ RUNX1, t(5;14)(q31;q32); IL3-IGH@ and $\mathrm{t}(1 ; 19)$ (q23;p13.3); TCF3-PBX1. Recognition of these genetic aberrations provides powerful independent prognostic information to the pediatric patient with B-lymphoblastic leukemia. 
In recent years, a variety of additional recurring genetic abnormalities in B-lymphoblastic leukemia have been recognized (eg RUNX1 amplification, $\mathrm{t}(17 ; 19)(\mathrm{q} 23 ; \mathrm{p} 13)$ and $\operatorname{dic}(9 ; 20)(\mathrm{p} 13 ; \mathrm{q} 11)) .^{2,3}$ Although these recurrent genetic aberrations are less common than those currently specified in the WHO 2008 classification, it is certain that some represent new distinct genetic subgroups of B-lymphoblastic leukemia. Recognition of newer subgroups generally requires longer patient accrual intervals given the low incidence of the particular genetic abnormality of interest, along with well-controlled and standardized treatment protocols, similar therapeutic regimens and accurate outcome assessment.

One recurrent abnormality in B-lymphoblastic leukemia that has received increasing attention since the mid to late 1990s is the RUNX1 (a.k.a. AML1) amplification (chromosomal location 21q22), which is detected via fluorescence in situ hybridization (FISH) with a probe that spans the RUNX1 region. ${ }^{-16} R U N X 1$ is a genetic locus of particular interest as, as a transcription factor, it is involved in a variety of chromosomal translocations in several acute leukemia types (eg t(8;21)(q22;q22); RUNXI$R U N X 1 T 1$ in acute myeloid leukemia and $\mathrm{t}(12 ; 21)$ (p13;q22); ETV6-RUNX1 in B-lymphoblastic leukemia). RUNX1 point mutations also occur in myeloid malignancies. ${ }^{17}$ Per larger studies, the incidence of B-lymphoblastic leukemia with RUNX1 amplification among pediatric B-lymphoblastic leukemias is $\sim 2 \% .^{10,12,13}$ Interestingly, this genetic abnormality, thus far, appears confined to the pediatric and young adult population. ${ }^{11}$ At present, based on the published literature, B-lymphoblastic leukemia with $R U N X 1$ amplification is reported to have an increased risk of relapse and worse overall prognosis. ${ }^{2,13-15,18}$ Given these compelling data, which span a decade and reveal a possible new distinct genetic subgroup of B-lymphoblastic leukemia, we sought to identify and evaluate cases of B-lymphoblastic leukemia with $R U N X 1$ amplification seen at our institution since the year 2000 .

\section{Materials and methods}

\section{Case Selection}

The University of New Mexico cytogenetic files were searched from 2000 to 2009 for cases of RUNX1 amplification in B-lymphoblastic leukemia (a.k.a. precursor B-cell acute lymphoblastic leukemia). Eight total cases were identified with sufficient clinical, laboratory, immunophenotypic and treatment data to warrant inclusion in the study. Age at diagnosis, gender, presenting white blood cell count, percent circulating blasts, bone marrow morphology and blast count, cerebrospinal fluid involvement, flow cytometric, FISH, cytogenetic, initial treatment protocol and follow-up data were collected. In all, 25 random consecutive cases of
B-lymphoblastic leukemia without RUNX1 amplification were identified as 'controls' for comparative analysis.

\section{Fluorescence In Situ Hybridization}

The RUNX1 amplification is identified using the LSI TEL/AML1 (ETV6/RUNX1) probe kit from Vysis (Abbott Molecular). This kit contains a probe spanning the RUNX1 genetic region (21q22), which is labeled with spectrum orange and a probe spanning the ETV6 region (12p13), which is labeled with spectrum green. FISH analysis was performed in all cases on bone marrow-fixed interphase cell suspensions. Cases exhibiting a RUNX1 amplification show variably sized clusters of orange signals (reflecting an intrachromosomal expansion) with ranges in signal copy numbers from 5 to $>15$ / interphase cell. Cases of RUNX1 polysomy are distinctly different from RUNX1 amplification as they show extra copies of chromosome 21. Polysomy shows additional orange signals per interphase cell; however, the signals are not clustered together but rather individually distributed throughout the cell.

\section{Cytogenetics}

For cytogenetic flask suspension cultures, an appropriate amount of bone marrow is placed in $10 \mathrm{ml}$ of RPMI 1640 media (Gibco) supplemented with L-glutamine, fetal bovine serum and antibiotics. Overnight and $48 \mathrm{~h}$ unstimulated cultures were set up. These flask cultures were harvested and G banded using standard techniques. Karyotype designation was made in accordance with the 2009 International System for Human Cytogenetic Nomenclature. ${ }^{19}$

\section{Flow Cytometry}

Flow cytometric immunophenotyping was performed in six of eight cases at our institution on bone marrow samples according to standard procedures. ${ }^{20}$ In brief, four-color or six-color flow cytometry was performed on a FACS Calibur or FACS Canto, respectively (Becton Dickinson Biosciences, San Jose, CA, USA). We evaluated the bone marrow specimens with antibodies against CD3, CD4, CD5, CD7, CD8, CD19, CD20, CD10, CD13, CD33, CD34, CD117, TdT, CD45, surface kappa, surface lambda, CD79a and CD22. Flow cytometry data were analyzed with Paint-a-Gate or Diva software (Becton Dickinson Biosciences). Positivity for a particular antigen is detected as a shift $>20 \%$ compared with the background normal cells.

\section{Treatment}

All patients were enrolled on Children's Oncology Group trials as appropriate for their year of diagnosis 
and available open protocols. The Pediatric Oncology Group 9900 series was open from 1999 to 2005. The AALL00232/331 series was open thereafter.

\section{Statistical Methods}

Fisher's exact and Wilcoxon rank-sum tests were used to compare the 8 cases of B-lymphoblastic leukemia with $R U N X 1$ amplification to 25 pediatric B-ALL without $R U N X 1$ amplification control cases. The tests were performed using Stata version 11.1. Gender, age, WBC count, percent circulating blasts, central nervous system disease and blast immunophenotypic profile were compared. A comparison of overall survival was not performed given the small sample size.

\section{Results}

We identified eight cases of pediatric B-lymphoblastic leukemia with RUNX1 amplification. Table 1 summarizes the clinical, laboratory, immunophenotypic, genetic and follow-up data in all eight patients as known. No adult cases were identified by our search strategy as the FISH probe to detect the cytogenetically cryptic ETV6-RUNX1 fusion (result of $t(12 ; 21)$ ) is typically only performed in childhood B-lymphoblastic leukemia cases.

\section{Clinical, Laboratory, Morphologic and Treatment Features}

We found the incidence of pediatric B-lymphoblastic leukemia with RUNX1 amplification to be $2 \%$ at our institution from January 2000 to December 2009. There was a male predominance $(\mathrm{M}: \mathrm{F}$ of $7: 1)$ with a median age at presentation of 9.5 years (range 3-15). Compared with controls, the older age at presentation was statistically significant $(P$-value $=0.006$, 3 years versus 9.5 years), while the male predominance was marginally significant $(P$-value $=0.082)$. The majority of patients presented with typical features of acute leukemia and bone marrow failure including bone pain, fatigue and easy bruising.

Peripheral white blood cell counts were overall low or normal (median $2.15 \times 10^{9} /$, range 1.7 $13.7 \times 10^{9} / \mathrm{l}$ ) in the B-lymphoblastic leukemia with RUNX1 amplification cases compared with 25 controls (median $4.7 \times 10^{9} / \mathrm{l}, P$-value $=0.081$ ). The percent circulating blasts was also low (median $11 \%$, range $0-24 \%$ ), although not statistically significant compared with controls (median 24\%, $P$-value $=0.110)$. Cytopenias were present in seven of eight patients, most commonly anemia and thrombocytopenia. Seven of eight cases presented with extensive iliac crest bone marrow involvement by B-lymphoblastic leukemia $(>85 \%$ blasts by morphology). Patient \#1 presented with bone pain and leukemia was diagnosed on a radiologically

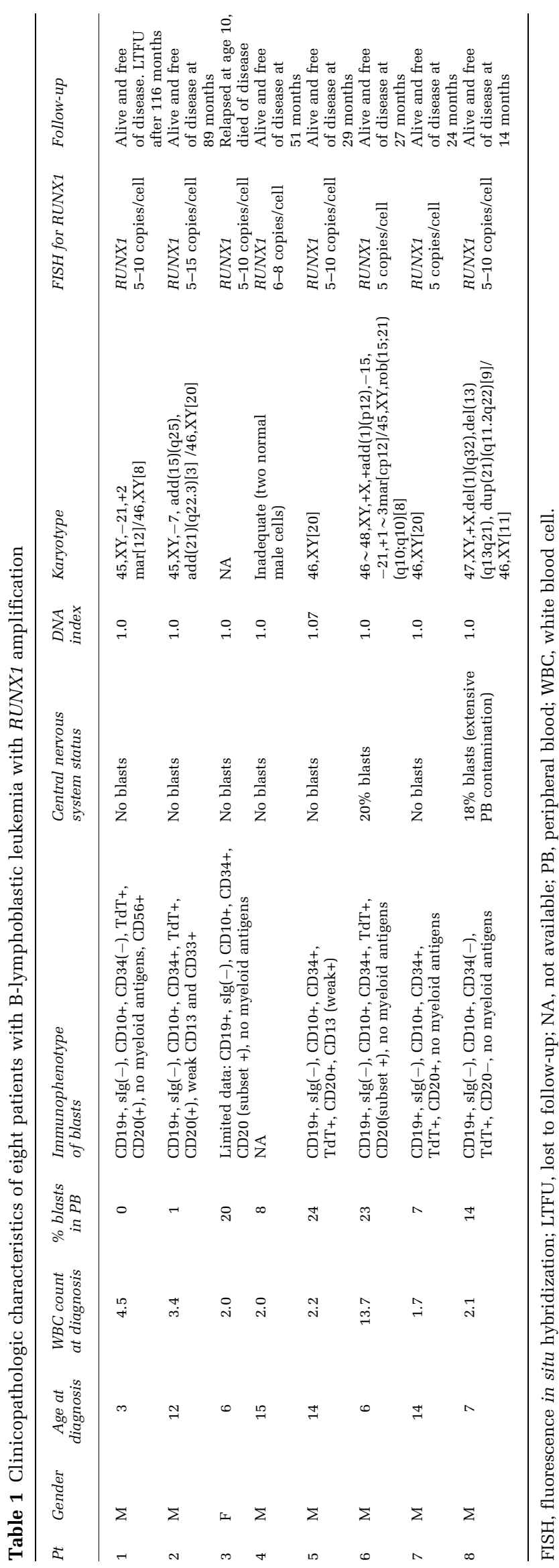


guided bone biopsy. The morphology of the lymphoblasts was typical in the majority of cases (five of seven): small to intermediate, dispersed yet somewhat dense chromatin, inconspicuous nucleoli and scant cytoplasm (French-American-British L1 type). Two of the cases demonstrated a mixture of slightly larger lymphoblasts with more cytoplasm and a variably prominent nucleolus (French-AmericanBritish L2) and L1 type lymphoblasts.

Two of eight patients $(25 \%)$ demonstrated involvement of the central nervous system via lymphoblasts in the cerebrospinal fluid. The presence of central nervous system positivity was not statistically significant $(P$-value $=0.999)$.

All patients were initially enrolled on a Children's Oncology Group protocol at the time of their diagnosis. Seven of eight patients $(88 \%)$ are alive and free of disease (mean 50 months, range 14-116 months). Patient \#3 presented in relapse and died of her disease.

\section{Flow Cytometric Features}

In the seven cases of B-lymphoblastic leukemia with RUNX1 amplification with flow cytometric data, we identified a typical B-lymphoblast immunophenotypic profile: CD19+, TdT + , CD10 +, surface immunoglobulin(-), CD79a + and cCD22 +. CD34 was positive in five of seven cases. Six of seven cases showed complete or subset CD20 positivity and two of seven cases demonstrated expression of myeloidassociated antigens (eg CD13 and CD33). Although a high proportion of cases expressed CD20, this finding, compared with controls, was only marginally significant $(P$-value $=0.088)$. Myeloid antigen expression by the lymphoblasts was not statistically significant $(P$-value $=0.999)$.

\section{FISH and Cytogenetic Features}

All eight cases of B-lymphoblastic leukemia with RUNX1 amplification show a typical clustering of $R U N X 1$ signals (orange) FISH pattern (Figure 1). The number of copies of the RUNX1 locus per cell ranged from 5 to 15 (Table 1). This RUNX1 amplification pattern was observed in $>80 \%$ of interphase cells indicating that it was present in all of the bone marrow lymphoblasts and not just a subset. Of the eight cases, four showed an abnormal karyotype, two showed a normal karyotype, one had insufficient metaphases for adequate analysis and one had no data available (Table 1). Of the four cases with an abnormal karyotype, the additional chromosome 21 material (RUNX1 genomic region) was seen as duplicated material on chromosome 21 (Figure 2) or on marker chromosomes. FISH for RUNX1 on metaphase cells from patients \#2 and \#8 confirmed that the amplified RUNX1 material resides on the cytogenetically abnormal chromosome 21 (data not shown). Although two of our cases were karyo-
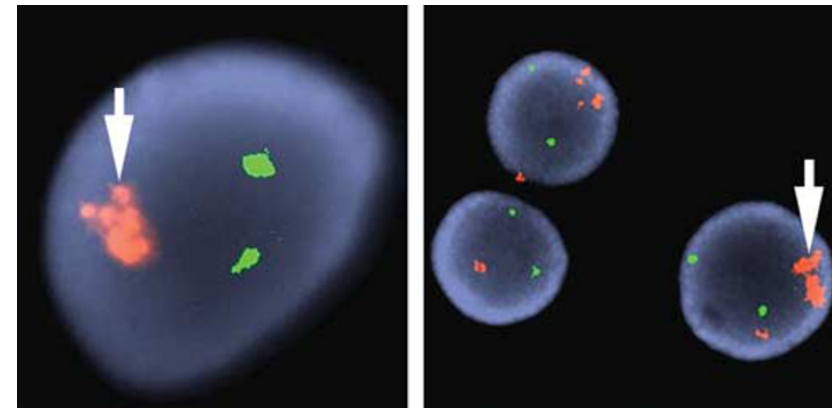

Figure 1 RUNX1 amplification in B-cell acute lymphoblastic leukemia. RUNX1 amplification is seen as variably sized clusters of orange signals (reflecting an intrachromosomal expansion) with ranges in signal copy numbers from 5 to $>15$ /interphase cell (arrows). The RUNX1 amplification is identified using the LSI TEL/AML1 (ETV6/RUNX1) FISH probe kit from Vysis (Abbott Molecular). This kit contains a probe spanning the RUNX1 genetic region (21q22), which is labeled with spectrum orange and a probe spanning the ETV6 region (12p13), which is labeled with spectrum green.

typically normal, it is unlikely that the $R U N X 1$ amplification is seen in an otherwise normal karyotypic background and may reflect the fact that the tumor cells did not grow in those instances. In fact, our patient \#5 clearly had an abnormal DNA index yet a normal conventional karyotype (Table 1).

\section{Discussion}

We report detailed clinicopathologic features of eight cases of pediatric B-lymphoblastic leukemia (a.k.a. precursor B-cell acute lymphoblastic leukemia) with the unique and recurrent genetic abnormality, RUNX1 amplification. The clinical and laboratory findings at disease presentation in our small cohort of patients with pediatric B-lymphoblastic leukemia with RUNX1 amplification are similar to those in the published literature.

Pediatric B-lymphoblastic leukemia with RUNX1 amplification was originally reported in the mid 1990s and has become increasingly recognized since that time. ${ }^{2-16} R U N X 1$ amplification is identified by FISH in interphase cells as a distinctive cluster of additional signals ( 5 to $>15$ signals/interphase nucleus) using a probe spanning the RUNX1 region (Figure 1). Most studies require at least five colocalized RUNX1 signals by interphase FISH to be considered RUNX1 amplified. ${ }^{14}$ In karyotypes, the RUNX1 amplification may be seen as an intrachromosomal amplification on chromosome 21 or as multiple tandem copies on a marker chromosome, a ring chromosome and double minutes (Figure 2). ${ }^{10,12}$ When there is expansion of chromosome 21 (eg dup(21q)), clear-cut determination of amplification of the RUNX1 region cannot be reliably performed by morphology alone and FISH is needed. ${ }^{12} \mathrm{~A}$ variety of cytogenetic alterations may be seen in addition to the RUNX1 amplification, including 


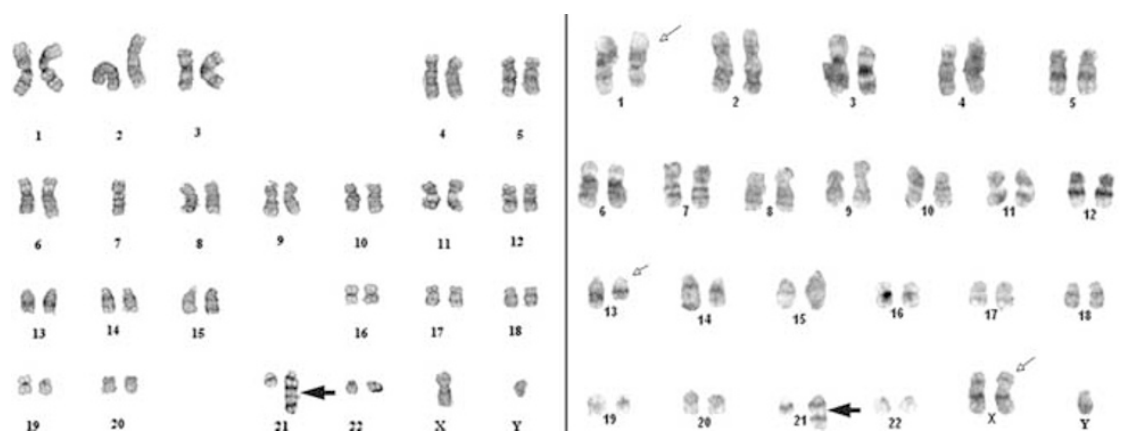

Figure 2 G-banded karyograms showing two examples of dup(21) in B-cell acute lymphoblastic leukemia with RUNX1 amplification. The normal chromosome 21 is on the left, and the abnormal dup(21) on the right (thick black arrows). Note the presence of additional unrelated cytogenetic abnormalities as well.

Table 2 Published studies with large number of pediatric ${ }^{a}$ patients with B-lymphoblastic leukemia with $R U N X 1$ amplification

\begin{tabular}{|c|c|c|c|c|c|c|}
\hline Reference & $\begin{array}{c}\text { Year } \\
\text { published }\end{array}$ & $\begin{array}{l}\text { Accrual years } \\
\text { for study } \\
\text { patients }\end{array}$ & $\begin{array}{l}\text { Incidence } \\
\text { of RUNX1 } \\
\text { amplification }\end{array}$ & $\begin{array}{l}\text { \# patients } \\
\text { reported } \\
\text { in study }\end{array}$ & $\begin{array}{l}\text { Treatment protocol } \\
\text { (if known) }\end{array}$ & Key findings \\
\hline $\begin{array}{l}\text { Harewood } \\
\text { et al }\end{array}$ & 2003 & NA & $1.5 \%$ & 13 & $\begin{array}{l}\text { UKALL XI, ALL97, } \\
\text { UKALL XII }\end{array}$ & $\begin{array}{l}\text { The karyotypic appearance of } R U N X 1 \\
\text { amplification may be seen as a ring, } \\
\text { duplication } 21 \text { or marker chromosome }\end{array}$ \\
\hline $\begin{array}{l}\text { Soulier } \\
\text { et al }\end{array}$ & 2003 & NA & NA & 16 & $\begin{array}{l}\text { FRALLE } 1993 \text { and } 2000 \text {, } \\
\text { EORTC } 58981 \text {, St Jude } \\
\text { Total XII and XIII, } \\
\text { BFM } 1995 \text { and } 1998\end{array}$ & $\begin{array}{l}R U N X 1 \text { amplification within pseudodiploid } \\
\text { or low hyperdiploid is an emerging genetic } \\
\text { subgroup of B-lymphoblastic leukemia }\end{array}$ \\
\hline $\begin{array}{l}\text { Robinson } \\
\text { et al }\end{array}$ & 2003 & NA & $1.5 \%$ & 8 & $\begin{array}{l}\text { UKALL XI, ALL97, } \\
\text { UKALL XII }\end{array}$ & $\begin{array}{l}R U N X 1 \text { amplification is associated with } \\
\text { a poor outcome }\end{array}$ \\
\hline $\begin{array}{l}\text { Harrison } \\
\text { et al }\end{array}$ & 2005 & 1997-2002 & $2 \%$ & 38 & ALL97 & $\begin{array}{l}\text { Interphase FISH using the ETV } 6 \text { and } \\
\text { RUNX1 probes is complementary to } \\
\text { conventional cytogenetics }\end{array}$ \\
\hline $\begin{array}{l}\text { Moorman } \\
\text { et al }\end{array}$ & 2010 & $1997-2002$ & $2 \%$ & 29 & ALL97 and 99 & $\begin{array}{l}\text { RUNX1 amplification is associated with } \\
\text { higher risk of relapse and death }\end{array}$ \\
\hline
\end{tabular}

${ }^{\mathrm{a}}$ Pediatric is defined as 1-18 years of age.

FISH, fluorescence in situ hybridization.

gain of an $\mathrm{X}$ chromosome and structural abnormalities of chromosomes $7,8,12,15$ and $16 .{ }^{12}$ We observed the gain of an X chromosome in 2 cases, as well as numerical abnormalities of 7 and 15, and structural aberrations of 1p12, 1q32, 13q and 15q.

To date, several large studies have been published that catalog the clinicopathologic, genetic and prognostic features of B-lymphoblastic leukemia with RUNX1 amplification (Table 2). Similar to our study, the incidence of B-lymphoblastic leukemia with RUNX1 amplification is estimated in $\sim 2 \%$ of pediatric B-lymphoblastic leukemias. In addition, we confirm an older age at presentation and a trend toward male predominance and low white blood cell count at disease presentation. ${ }^{12}$ Neither expression of surface CD20 or myeloid antigens on the blasts nor involvement of the cerebrospinal fluid reached statistical significance in our cohort. In our small cohort of patients, seven of the eight patients $(88 \%)$ are alive and free of disease (mean 50 months, range 14-116 months). In published series, pediatric B-lymphoblastic leukemia with RUNX1 amplification has been associated with an increased risk of relapse and worse overall prognosis. ${ }^{2,13-15,18}$ In addition, very recent unpublished data from the Children's Oncology Group also suggests that there are inferior outcomes of children with B-lymphoblastic leukemia with RUNX1 amplification compared with those without RUNX1 amplification with lower intensity post-induction therapy (written communication from the Children's Oncology Group provided to the authors by Stuart Winter, MD).

The RUNX1 gene encodes a transcription factor that has an essential role in definitive hematopoiesis. ${ }^{21}$ Unlike B-lymphoblastic leukemia with RUNX1 amplification, RUNX1 is likely better known as a translocation partner in acute myeloid leukemia with RUNX1-RUNX1T1 fusion (t(8;21;q22;q22)) or in B-lymphoblastic leukemia with ETV6-RUNX1 fusion (t12;21)(p13;q22). Alternative abnormalities in the RUNX1 gene include point mutations, which are frequently identified in acute leukemias. ${ }^{17}$ Similar to the amplification described herein, RUNX1 amplification has also been reported in acute myeloid leukemia and myelodysplastic syndrome. ${ }^{22}$ It is proposed that the intrachromosomal amplification of RUNX1 may 
arise from a breakage-fusion-bridge cycle. ${ }^{23}$ In brief, this mechanism entails an initial break in doublestranded DNA, formation of a dicentric chromosome and the ultimate generation of a chromosome with an inverted repeat sequence. Subsequent mitotic cycles result in the addition of multiple copies of these inverted repeat sequences (amplification). While it is evident that the RUNX1 protein has a key leukemogenic role in multiple neoplastic hematopoietic disorders, the specific role of RUNX1 amplification currently remains unknown.

In conclusion, pediatric B-lymphoblastic leukemia with $R U N X 1$ amplification is a rare entity that can be readily identified at diagnosis with a $R U N X 1$ FISH probe. The patient population shows a male predominance $(P$-value $=0.082)$ and older age at presentation (median 9.5 years, $P$-value $=0.006$ ) Although pediatric B-lymphoblastic leukemia with $R U N X 1$ amplification has a low incidence $(2 \%)$ compared with other B-lymphoblastic leukemia genetic subgroups, additional larger studies are needed to evaluate the independent prognostic significance of RUNX1 amplification with the ultimate goal being the identification of the optimal therapeutic regimen for these children.

\section{Disclosure/conflict of interest}

The authors declare no conflict of interest.

\section{References}

1 Borowitz MJ, Chan JKC. B lymphoblastic leukaemia/ lymphoma with recurrent genetic abnormalities In: Swerdlow SH, Campo E, Harris NL et al (eds). WHO Classification of Tumours of Hematopoietic and Lymphoid Tissues. IARC: Lyon, France, 2008.

2 Moorman AV, Ensor HM, Richards SM, et al. Prognostic effect of chromosomal abnormalities in childhood B-cell precursor acute lymphoblastic leukaemia: results from the UK Medical Research Council ALL97/ 99 randomised trial. Lancet Oncol 2010;11:429-438.

3 Harrison CJ, Haas O, Harbott J, et al. Biology and Diagnosis Committee of International Berlin-FrankfürtMünster study group. Detection of prognostically relevant genetic abnormalities in childhood B-cell precursor acute lymphoblastic leukaemia: recommendations from the Biology and Diagnosis Committee of the International Berlin-Frankfürt-Münster study group. Br J Haematol 2010;151:132-142.

4 Le Coniat M, Romana SP, Berger R. Partial chromosome 21 amplification in a child with acute lymphoblastic leukemia. Genes Chromosomes Cancer 1995;14: 204-209.

5 Busson-Le Coniat M, Nguyen Khac F, Daniel MT, et al. Chromosome 21 abnormalities with AML1 amplification in acute lymphoblastic leukemia. Genes Chromosomes Cancer 2001;32:244-249.

6 Niini T, Kanerva J, Vettenranta K, et al. AML1 gene amplification: a novel finding in childhood acute lymphoblastic leukemia. Haematologica 2000;85: 362-366.
7 Dal Cin P, Atkins L, Ford C, et al. Amplification of AML1 in childhood acute lymphoblastic leukemias. Genes Chromosomes Cancer 2001;30:407-409.

8 Mathew S, Rao PH, Dalton J, et al. Multicolor spectral karyotyping identifies novel translocations in childhood acute lymphoblastic leukemia. Leukemia 2001;15:468-472.

9 Morel F, Herry A, Le Bris MJ, et al. AML1 amplification in a case of childhood acute lymphoblastic leukemia. Cancer Genet Cytogenet 2002;137:142-145.

10 De Braekeleer E, Douet-Guilbert N, Morel F, et al. RUNX1 amplification in B-cell acute lymphoblastic leukemia. Leuk Lymphoma 2010;51:329-332.

11 Penther D, Preudhomme C, Talmant P, et al. Amplification of AML1 gene is present in childhood acute lymphoblastic leukemia but not in adult, and is not associated with AML1 gene mutation. Leukemia 2002;16:1131-1134.

12 Harewood L, Robinson H, Harris R, et al. Amplification of AML1 on a duplicated chromosome 21 in acute lymphoblastic leukemia: a study of 20 cases. Leukemia 2003;17:547-553.

13 Harrison CJ, Moorman AV, Barber KE, et al. Interphase molecular cytogenetics screening for chromosomal abnormalities of prognostic significance in childhood acute lymphoblastic leukaemia: a UK Cancer Cytogenetics Group Study. Brit J Haematol 2005;129:520-530.

14 Soulier J, Trakhtenbrot L, Najfeld V, et al. Amplification of band q22 of chromosome 21, including AML1, in older children with acute lymphoblastic leukemia: an emerging molecular cytogenetic subgroup. Leukemia 2003;17:1679-1682.

15 Robinson HM, Broadfield ZJ, Cheung KL, et al. Amplification of AML1 in acute lymphoblastic leukemia is associated with a poor outcome. Leukemia 2003;17:2249-2250.

16 Pérez-Vera P, Montero-Ruíz O, Frías S, et al. Multiple copies of RUNX1: description of 14 new patients, follow-up, and a review of the literature. Cancer Genet Cytogenet 2008;180:129-134.

17 Osato M. Point mutations in the RUNX1/AML1 gene: another actor in RUNX leukemia. Oncogene 2004;23: 4284-4296.

18 Moorman AV, Richards SM, Robinson HM, et al. UK Medical Research Council (MRC)/National Cancer Research Institute (NCRI) Childhood Leukaemia Working Party (CLWP). Prognosis of children with acute lymphoblastic leukemia (ALL) and intrachromosomal amplification of chromosome 21 (iAMP21). Blood 2007;109:2327-2330.

19 Shaffer LG, Slovak ML, Campbell LJ (eds). ISCN 2009: an International System for Human Cytogenetic Nomenclature. Karger: Basel, 2009.

20 Rahemtullah A, Reichard KK, Preffer FI, et al. A doublepositive CD4+CD8+ T-cell population is commonly found in nodular lymphocyte predominant Hodgkin lymphoma. Am J Clin Pathol 2006;126:805-814.

21 Cohen Jr MM. Perspectives on RUNX genes: an update. Am J Med Genet A 2009;149A:2629-2646.

22 Moosavi SA, Sanchez J, Adeyinka A. Marker chromosomes are a significant mechanism of high-level RUNX1 gene amplification in hematologic malignancies. Cancer Genet Cytogenet 2009;189:24-28.

23 Robinson HM, Harrison CJ, Moorman AV, et al. Intrachromosomal amplification of chromosome 21 (iAMP21) may arise from a breakage-fusion-bridge cycle. Genes Chromosomes Cancer 2007;46:318-326. 